\title{
Trait Evolution in Adaptive Radiations: Modeling and Measuring Interspecific Competition on Phylogenies
}

\author{
Magnus Clarke, ${ }^{\star}$ Gavin H. Thomas, and Robert P. Freckleton \\ Department of Animal and Plant Sciences, University of Sheffield, Sheffield S10 2TN, United Kingdom \\ Submitted December 4, 2015; Accepted September 12, 2016; Electronically published December 7, 2016 \\ Online enhancements: appendixes, zip file. Dryad data: http://dx.doi.org/10.5061/dryad.3sk15.
}

\begin{abstract}
The incorporation of ecological processes into models of trait evolution is important for understanding past drivers of evolutionary change. Species interactions have long been thought to be key drivers of trait evolution. However, models for comparative data that account for interactions between species are lacking. One of the challenges is that such models are intractable and difficult to express analytically. Here we present phylogenetic models of trait evolution that include interspecific competition among chosen species. Competition is modeled as a tendency of sympatric species to evolve toward difference from one another, producing trait overdispersion and high phylogenetic signal. The model predicts elevated trait variance across species and a slowdown in evolutionary rate both across the clade and within each branch. The model also predicts a reduction in correlation between otherwise correlated traits. We use an approximate Bayesian computation approach to estimate model parameters. We find reasonable power to detect competition in sufficiently large $(20+$ species $)$ trees compared with Brownian trait evolution and with OrnsteinUhlenbeck and early burst models. We apply the model to examine the evolution of bill morphology of Darwin's finches and find evidence that competition affects the evolution of bill length.
\end{abstract}

Keywords: adaptive radiation, character displacement, phylogenetics, ecological modeling.

\section{Introduction}

There is an increasing drive to combine evolutionary and ecological perspectives in order to fully capture the longterm dynamics of ecological communities (Johnson and Stinchcombe 2007; Cavender-Bares et al. 2009; Schoener 2011; Pennell and Harmon 2013; Hadfield et al. 2014; Price et al. 2014; Pigot and Etienne 2015). This has led to insights into the roles of ecological processes - such as competitive exclusion and character displacement—in shap-

* Corresponding author; e-mail: mclarke3@shef.ac.uk. ORCIDs: Freckleton, http://orcid.org/0000-0002-8338-864X.

Am. Nat. 2017. Vol. 189, pp. 000-000. (C) 2016 by The University of Chicago. 0003-0147/2017/18902-56692\$15.00. All rights reserved. This work is licensed under a Creative Commons Attribution 4.0 International License (CC BY 4.0), which permits reuse of the work with attribution.

DOI: $10.1086 / 689819$ ing trait evolution and today's distributions of traits (Webb et al. 2002; Kraft et al. 2007; Emerson and Gillespie 2008; Vamosi et al. 2009). However, linking such patterns in data to underlying processes is difficult, since any given pattern could be the outcome of several processes (Dayan and Simberloff 2005; Mayfield and Levine 2010).

Evidence that competition has shaped trait evolution has been generated using two main approaches. The first is the observation of character displacement, that is, a tendency for species with overlapping ranges to exhibit increased phenotypic differences where they coexist (Schluter and McPhail 1992; Dayan and Simberloff 2005; Pfennig and Pfennig 2010; Stuart and Losos 2013). The second line of evidence for competitive effects makes use of a phylogeny to measure the distribution of species trait values relative to a null model (Webb et al. 2002; Freckleton and Harvey 2006; Vamosi et al. 2009). This is especially useful for adaptive radiations, where typically several similar species are confined to the same geographical area. Distributions that are more even than expected by chance (Webb et al. 2002; Dayan and Simberloff 2005; Davies et al. 2012) are taken as evidence that past competition caused species to seek unique ecological niches.

Convergent evolution of sets of species in separate clades has also been observed and interpreted as evidence of interspecific competition (Moen and Wiens 2009). With close niche packing, interspecific competition can reduce evolutionary rates, even with a changing environment (De Mazancourt et al. 2008). Phylogenetic comparative models of adaptive radiations have slowing rates of phenotypic evolution, implicitly assuming that competition for finite niche space is an underpinning mechanism (e.g., early burst model; Harmon et al. 2010a). Despite much study, however, the importance of competition remains uncertain (Gillespie et al. 2001; Cavender-Bares et al. 2009), and importantly, direct tests for evidence of past competition in phylogenetic data are lacking.

One approach could be to explicitly model the evolution of traits in systems of species in which competition is occurring. In general, evolutionary models use some combination 
of continuous random change through time (Felsenstein 1973), possibly with changes of rate (Garland et al. 1992; Pagel 1997; Freckleton et al. 2002; Blomberg et al. 2003; Eastman et al. 2011; Revell et al. 2012; Thomas and Freckleton 2012), discrete random changes at speciation events (Ingram 2010), or shifts in shared adaptive optima (Uyeda and Harmon 2014). However, phylogenetic models of trait evolution are ecologically neutral, since they are stochastic models that depend on the independent evolution of each species to be statistically well behaved (Pennell and Harmon 2013). Processes such as competition between species are typically not accounted for. In previous models, species interactions have been assumed to generate phenomenological outcomes. For example models may assume rate slowdowns associated with competition among lineages either implicitly by modeling through time (Harmon et al. 2010a) or explicitly (Mahler et al. 2010). Several models include clade-wide nonrandom effects (Hansen 1997; Price 1997; Harvey and Rambaut 2000; Freckleton and Harvey 2006; Bartoszek et al. 2012), reflecting the interaction of species with their environments, but none of these models permits trait values to be influenced by interspecific interactions.

Phylogenetic data sets have been simulated with competitive interactions (Freckleton et al. 2003; Nuismer and Harmon 2015). However, direct parameterization with data is difficult because of the complexity of accounting for interspecific interactions. Niche-filling models of trait evolution on trees (Price 1997; Harvey and Rambaut 2000; Freckleton and Harvey 2006) are models of adaptive radiations where new species move discretely to the nearest of a random set of points (niches) in trait space. Simulations under these models show that such ecological processes affect inferences drawn from comparative analyses. The most important conclusion from the analysis of such models is that methods based on Brownian motion are inappropriate or even misleading when applied to traits evolving in such systems. However, the problem of modeling such data has never been satisfactorily resolved (Freckleton and Harvey 2006), largely because of the complexity of statistically describing the traits of a set of interacting species.

In terms of fitting complex models to data, one potential approach is approximate Bayesian computation (ABC; see Beaumont 2010). This provides a simple method for generating posterior probabilities of models, provided we can simulate them. It is therefore well suited to fitting complex models, where it is not possible to compute a likelihood function. In this way, species interactions could be incorporated into evolutionary models, thus permitting better inference of the ecology underlying trait evolution. Processes such as character displacement and mutualism affect trait values, and $\mathrm{ABC}$ is a means of comparing models that explicitly include these processes. $\mathrm{ABC}$ has been explored for simple phylogenetic trait evolutionary models (Kutsu- kake and Innan 2013), including birth-death models (Slater et al. 2012), but its flexibility has not previously been used for including complex effects like interspecific interactions.

In this article, we introduce a new model for the evolution of interacting species within phylogenetic data. Our objective is to create a model that includes characterdisplacement interactions and makes realistic predictions but that also may be fitted to real data. We do not assume that all species are interacting with each other but instead allow competitive interactions to be turned on and off at different times and for different pairs of species. We use this flexibility in two ways. First, interactions can be turned on for sympatric species and turned off for allopatric species, so that the model can encompass large groups of species with variously overlapping or nonoverlapping ranges. Second, we can add a delay, after each new species arises, before it begins interacting with the other species. This corresponds to a scenario where speciation occurs in allopatry and a subsequent range expansion brings the new species back into contact after a significant time interval. These two uses for optional sympatry can of course be combined.

There are two main diffusion models of trait evolution: Brownian motion (BM; Felsenstein 1973, 1985) and the Ornstein-Uhlenbeck (OU) model (Hansen 1997). The OU model is based on BM, with the addition of an overall optimum trait value to which all lineages are attracted. The strength of attraction adds a further parameter. However, we chose to build our model on the BM model. The BM model is a very simple, neutral model, while the OU model produces, in some respects, results that are the opposite of those produced by our competition model. The ability to reject BM in favor of either OU or competition may therefore be a useful aid in interpreting data.

The model predictions are compared with those of the $\mathrm{BM}$ and rate change models for sympatric clades. We then outline how ABC methods may be used to detect competition effects, and we show that the model is readily fitted to data. Finally, we apply these methods to a simple case study, the adaptive radiation of Galapagos finches.

\section{Methods \\ The Model}

Under the BM model of trait evolution (Felsenstein 1973), for each species $i$, a trait value $x_{i}$ evolves according to the differential equation

$$
\mathrm{d} x_{i}=\sigma \mathrm{d} W_{i}(t),
$$

where $W(t)$ is the integral of the continuous white noise function, such that over a finite time it has a normal probability distribution: $W(t) \sim N(0, \Delta t)$. The BM model has two free parameters, the evolutionary rate $\sigma$ and the root trait 
value $x_{\text {root }}(0)$. The expected variance between tips is proportional to the branch length separating them.

Many models for comparative data are based on modifying this model by adding additional parameters (Pagel 1997; Blomberg et al. 2003; Eastman et al. 2011; Revell et al. 2012; Thomas and Freckleton 2012; Boucher and Démery 2016). For example, exponential rate change models replace $\sigma$ with $g^{-t} \sigma$ (Blomberg et al. 2003), so that $g<1$ corresponds to an increasing rate of trait evolution and $g>1$ means a decreasing rate (an evolutionary slowdown). Notably, these models typically assume that the evolutionary trajectories of species traits are independent and assume that there are no interactions between different species.

Our competition model is based on the BM model, with a term added to account for interspecific interactions. Competition is modeled such that species with similar trait values tend to evolve away from each other, while species with dissimilar trait values have little influence on each other. To achieve this, we assume a flat fitness surface for trait values in the absence of other species. In effect, we assume that if the trait in question has a one-to-one correspondence with some resource (e.g., body size and prey size), then the distribution of resources is flat. We assume that a species with a given trait value has a corresponding ideal resource but also uses up other resources such that the distribution of resource types used is normal and centred on the ideal resource type. Therefore, a Gaussian curve is associated with each species along a single trait axis representing this resource use and consequently its amount of influence on other species as a function of the difference in trait value between the two species (Doebeli and Dieckmann 2003; Leimar et al. 2008, 2013; Pigolotti et al. 2010).

The repulsion between two species in trait space is assumed to be proportional to the overlap of each of their associated curves. For the evolution of a single trait $x$ in a species $i$, we obtain a deterministic term, scaled by a parameter $\alpha$ :

$$
\mathrm{d} x_{i}=a \sum_{j} S_{i j}(t) \operatorname{overlap}\left(x_{i}, x_{j}\right) \mathrm{d} t+\sigma \mathrm{d} W_{i}(t) .
$$

The area of overlap of two normal curves is related to the cumulative normal function $\Phi$ of minus the distance (in standard deviations) between them, such that the overlap is equal to $2 \Phi$ (-distance/2) (Inman and Bradley 1989). The overlap of two curves very far from each other is $2 \Phi(-\infty)=0$, whereas the overlap of two curves with the same center is $2 \Phi(0)=1 . \mathrm{S}$ is a sympatry matrix, with elements $S_{i j}$ each equal to either 0 or 1 . If species $i$ and $j$ inhabit the same geographical area and have the opportunity to interact, then we can set $S_{i j}=1$; otherwise, $S_{i j}=0$. The matrix $\mathbf{S}$ can be a function of time, permitting any given pair of species to spend time effectively in allopatry and in sympatry.
The relative intensity of competition is measured by the value of the competition parameter $a$ relative to the Brownian rate parameter $\sigma$. Ideally, we would have chosen to make the kernel width an additional parameter of the model. However, in practical terms, it would not have been possible to distinguish this effect from that of the competition parameter $a$. Appendix A shows that to a linear approximation the effects of the two are the same, and so they are likely to be statistically indistinguishable.

The instantaneous change of the trait value $x_{i}$ of species $i$ is given by

$$
\mathrm{d} x_{i}=a \sum_{j} S_{i j}(t) e_{i j} 2 \Phi\left(-\left|x_{i}-x_{j}\right|\right) \mathrm{d} t+\sigma \mathrm{d} W_{i}(t) .
$$

Each $x_{j}$ is a vector in trait space; the index $j$ denotes species. The right-hand side has two terms: the first is a deterministic competition term, which pushes apart species that are nearby in trait space; $e_{i j}$ is the unit vector pointing from species $j$ to species $i$ in trait space. Thus, $a e_{i j} \Phi\left(-\left|x_{i}-x_{j}\right|\right)$ is a vector in trait space pointing from species $i$ to species $j$, proportional to the model parameter $a$ and depending on the closeness in trait space of species $i$ and $j$. What distinguishes this model from previous ones is that in the competition term of the equation, all traits are linked: the evolution of two species away from each other in trait space depends on the Euclidian distance between them as well as their distances to all other species. We largely concentrate here on single resources and traits. However, more generally, a multivariate normal curve in trait space may be associated with each species in order to model interactions along several resource axes.

In both the BM and the competition models, trait variance increases without bound as time progresses. In reality, there are limits that will be driven by ecology or by developmental and physiological constraints. We therefore adapted the model by imposing hard limits on trait space, such that species can evolve up to a chosen extreme value but no further. This model was simulated alongside the limitless model; hence, we obtain a new model with constrained trait/ niche space. We assume that the limits are symmetric about the root trait value and equal to the most extreme value $L$.

\section{Simulation Framework}

In diffusion models such as BM or OU, trait evolution may be modeled readily and quickly because species are assumed to be independent. However, our model requires that we simulate evolution over interacting branches, which makes it far more computationally demanding. The approach we used was to take a discrete approximation to the continuous differential process in equation (3). A large number of time steps were used, and trait values were computed 
sequentially for each step using the discrete approximation to equation (3):

$$
\Delta x_{i}=a \sum_{j} S_{i j}(t) e_{i j} 2 \Phi\left(-\left|x_{i}-x_{j}\right|\right) \Delta t+\sigma W(\Delta t),
$$

where $W(\Delta t) \sim N(0, \Delta t)$, that is, a normal distribution with mean 0 and variance $\Delta t$. For every time step, pairwise trait value differences must be computed between all species, giving $\left|x_{i}-x_{j}\right|$ for all species $i$ and $j$. Then, the trait values are all updated according to equation (4). For a tree with 20 tips divided into 1,000 time steps, a typical modern consumer processor takes about 0.008 seconds to complete a simulation. The simulation of traits on a single clade is single threaded.

Simulations were performed on random ultrametric trees generated under a Yule process (TESS; Hoehna 2013) with between two and 100 tips. These tree sizes are large enough to demonstrate the effects of competition and provide a range over which our power to detect competition varies from low to high. Because the competition model is designed for sympatric, interacting sets of species having undergone adaptive radiation, it is unlikely that numbers of species will be very large. For example, the average clade size in Harmon et al.'s $(2010 a, 2010 b)$ survey of adaptive radiations was 30 tips. We first estimated some summary statistics for a single 10-tip tree: the fitted Brownian rate $\sigma$, phylogenetic signal $K$ (Blomberg et al. 2003), and the rate change parameters $\kappa$ and $\delta$ (Pagel 1997). We did this for a range of competition strengths from $a=0$ to $a=5$, recording trait values through time to generate figure 1 . We also repeated this with competition and limits and with the two main subclades set to be allopatric (noninteracting) with one another.

To compare tip trait value distributions, we generated a single 40-tip random tree and collected trait data from many simulations under each of three evolutionary regimes: BM, competition, and competition with limits. The distributions for each regime included all the corresponding data sets. To assess the accumulation of trait variance, we did the same again but with two different values of the competition parameter $a$.

Tree size has an effect on the variance of tip trait values in our competition model, unlike BM. To assess this, we generated trees that had numbers of tips from five to 100 . For each possible number of tips within this interval, we generated 50 random trees. For each tree, we then simulated a trait data set under BM, competition, and competition with limits. The mean tip trait value variance was plotted as a function of the number of tips.

Competition also affects correlations between pairs of coevolving traits. We simulated traits whose Brownian evolution was strongly correlated ( $80 \%$ weighting for a single Brownian process and a $20 \%$ weighting for additional inde- pendent Brownian processes; under pure $\mathrm{BM}$, this results in a correlation of tip trait values of approximately $r^{2}=$ 0.8 ) under a wide range of competition strengths ( $a=0$ to $a=5)$. We did this for a single small tree (10 tips) and a single larger tree (40 tips). We also repeated these steps for four fixed values of $a(0,1,4,8)$ while varying the BM dependence (i.e., the amount of shared change between traits) and observed the resultant correlation between tip trait values.

The sympatry matrix $S(t)$ in equations (2) and (3) allows us to control which lineages interact and when. This means we can set up simulations where lineages start to have competitive interactions a fixed time after they start. This corresponds to the scenario of speciation in allopatry and subsequent range expansion so that all lineages eventually come back into contact. We simulated data with a range of delay periods $(0.1,0.2,0.5$, and 1.0 times the mean time between speciation events) and generated power estimates for detecting competition in these cases. We also generated an example data set-with a delay period of 0.5 times the mean time between speciation events - on a single 16-tip tree for comparison with other models (fig. $1 E$ ). It is important to note that we do not fit our sympatry matrix to data. The sympatry or allopatry of any given set of species is assumed to be known from independent data on species' ranges. A delay between speciation and competitive interactions can also be added, but again, this is preset by the user, not fitted.

The reasons for basing the competition model on BM rather than the OU model are detailed in the introduction. However, for the sake of comparison, we did run some simulations of an OU + competition model. The model is detailed in appendix B, and an example simulation is shown in figure $1 D$.

Finally, we assessed the effect of competition on phylogenetic signal. A single 100-tip tree was generated, and trait values were simulated for a range of competition parameter strengths ( $a=0$ to $a=5$ ). Blomberg's $K$ (Blomberg et al. 2003) was then computed using the Kcalc function in the R package picante (Kembel et al. 2010).

\section{Model Comparisons and Likelihoods}

We fitted the model to data using ABC (reviewed in Beaumont 2010; Csilléry et al. 2010; Hartig et al. 2011). ABC can be used for comparing the probabilities of data sets under different models when these probabilities are difficult to compute directly. This is because the only requirement to perform $\mathrm{ABC}$ is that we can simulate new data sets using the model. The data set probabilities are approximated by simulating a large number of data sets and accepting only those simulations that are very similar to the observed data set. This similarity can be judged either from the data values 

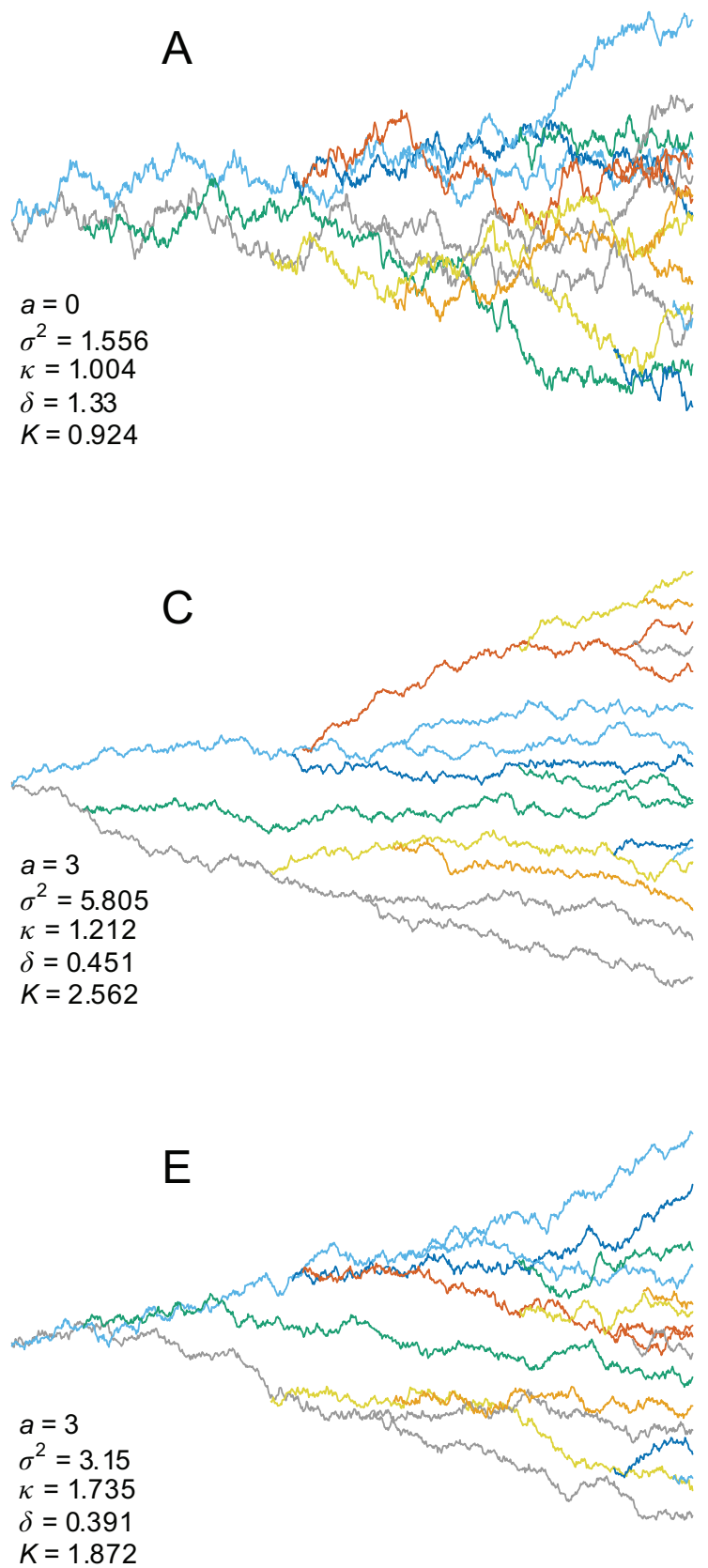
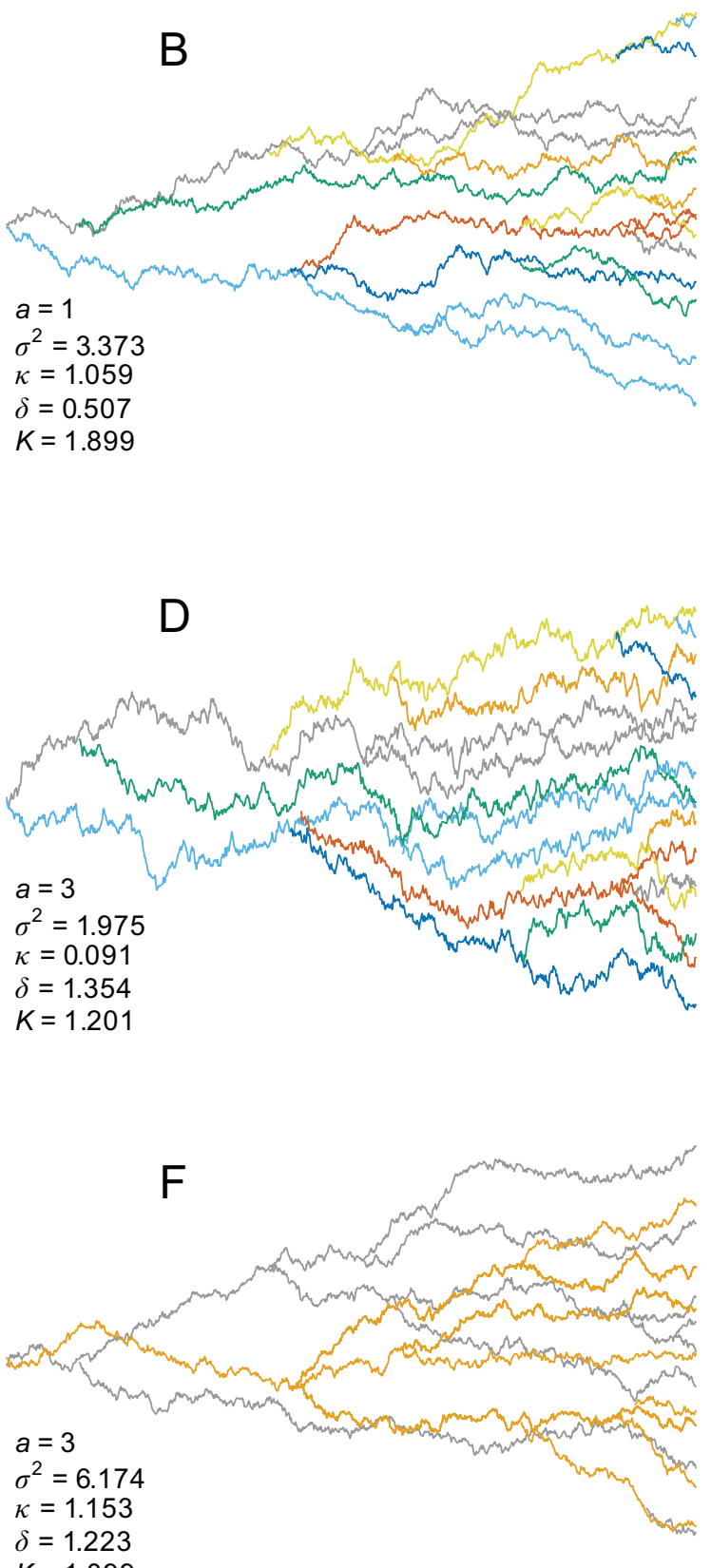

Figure 1: Examples of a single trait evolving under the competition model with different strengths of the competition effect parameter $a$. A single random 16-tip tree is used throughout. The Brownian motion (BM) parameter $\sigma=1$ is used for all the simulations. The parameter values listed by each plot are the estimates obtained using the data shown; these are subject to substantial stochastic variation between simulations. $A$, When $a=0$, we recover the BM model. $B$, Simulated using $a=1$. C, Simulated using $a=3$. D, Competition plus Ornstein-Uhlenbeck (OU) model, with the OU parameter $\alpha$ set to 5. Strong competition and OU effects cancel each other out in some respects. E, Delayed competition, where each new lineage starts to experience competitive interactions a fixed time after it appears. This corresponds to a scenario of allopatric speciation and subsequent range expansion. $F$, Here the two main subclades of the tree do not interact with each other, though there is strong competition within the subclades. This corresponds to a mixture of sympatric and allopatric species, for example, a radiation on two different islands. 
themselves or using summary statistics. The proportion of simulations that are accepted is then assumed to be proportional to the data set probability. When the model contains continuous parameters, we sample across these parameters and obtain an approximate probability density for the observed data under any point in a range of parameter values. This can be used to estimate the likelihood curves of fitted models. ABC relies on the likelihood being a fairly smooth function of the model parameters (Hartig et al. 2011). Since our model progressively changes the trait distribution and phylogenetic signal smoothly (with respect to the competition strength $a$ ), we expect the likelihood to be a smooth function.

To apply ABC to phylogenies (e.g., Beaumont 2010), we sample the parameters of the evolutionary model randomly many times from a prior parameter distribution. Here we choose the prior distribution to be uniform, with the model necessitating a hard limit at 0 for both the Brownian rate and the competition strength. For each set of parameters, trait data sets are then simulated for the known phylogeny. Summary statistics are generated for the simulated data, and only those simulations for which the summary statistics are within a small value $\varepsilon$ of the observed data's summary statistics are accepted. Thus, for observed data $D$ and tolerance $\varepsilon$, we accept some simulated data $D^{\prime}$ if

$$
\rho\left(D^{\prime}, D\right)<\varepsilon,
$$

where $\rho$ is the discrepancy - or distance in summary statistic space-between $D^{\prime}$ and $D$. In practice, we chose the tolerance $\varepsilon$ on the basis of the size of the posterior sample that we wanted to obtain, so we might simulate a million data sets and choose $\varepsilon$ such that we accept the best 500 simulations. By plotting acceptance rate against parameter values, we obtain an estimated likelihood surface.

To compare simulated and observed data sets, it is necessary to compare summary statistics. We chose to use three summary statistics: the mean and the variance of the differences between each species and its closest neighbor in trait space, and the overall phylogenetic signal as measured by Blomberg's $K$ (Blomberg et al. 2003). The rationale for using these three statistics was to capture the overall amount of evolution, the overdispersion of trait values, and the phylogenetic structuring of the trait values. There is no well-established procedure for choosing summary statistics for ABC. High sufficiency is needed to compare models, but the ABC method quickly loses accuracy and stability with large numbers of summary statistics (Csilléry et al. 2010). Our summary statistics were chosen on a pragmatic basis, since they capture the important aspects of the model's behavior, namely, increased divergence between sibling species and an even overall distribution of traits across the phylogeny.
We chose to compare the competition model with the BM model using maximum approximated likelihood, because the BM model is embedded in the competition model. The null and alternative $\mathrm{ABC}$ acceptance rates $A$ give an estimate of the likelihood $L(H \mid D)$ of the observed data set under the various model parameters. This assumes a smooth probability distribution with an overall peak and a separate peak on the $a=0$ (Brownian motion) plane. Since small changes to the model parameters should create small changes in average trait distributions, the assumption of smoothness should be met, provided that many simulations are used. The two peaks define the null and alternative model parameters. When there is no prior difference in model likelihood expectation, the log-likelihood ratio statistic for the comparison of two models $H_{0}$ and $H_{1}$ is given by

$$
-2 \log \frac{L\left(H_{1} \mid D\right)}{L\left(H_{0} \mid D\right)}=-2 \log \frac{A_{1}}{A_{0}} \text {. }
$$

When the models are nested, this test statistic approximates a $\chi^{2}$ distribution, given certain assumptions: large samples and normally distributed parameters. However, these assumptions may be significantly violated by phylogenetic methods (Freckleton 2009). For instance, in the BM model, $\sigma$ is bounded at 0 , and in our competition model, $a$ is also bounded at 0 . To correctly interpret the test statistic, therefore, the null distribution of the log-likelihood ratio test statistic was assessed with a parametric bootstrap.

The bootstrap was undertaken by performing the model comparison analysis on data sets generated under BM to create a null distribution of likelihood ratios. Then, if, for example, we want to know the likelihood ratio corresponding to a $P$ value of .05, we simply look at the ninety-fifth percentile of the null distribution. The resulting type I error rate is therefore chosen by design: if a significant likelihood ratio is one that corresponds to a $P$ value of $5 \%$, then the type I error rate is $5 \%$. To estimate typical significance thresholds, we performed this procedure for random trees, using 1,000 random data sets.

The power to reject BM in favor of the competition model was assessed by using random ultrametric Yule trees $(20,40$, 60 , and 80 tips). The bootstrap process was performed to determine the significance threshold for that tree. Then, for a given value of the competition parameter $a$, we simulated a large number of data sets and determined the likelihood ratio (between the BM and competition models) for each one. The proportion of these data sets that showed significant support for competition effects defines the power of the model for that value of $a$. We repeated this process for a range of competition strengths from $a=0$ to $a=5$. This range covers evolution from a Brownian process with no interspecific interaction $(a=0)$ to a largely deterministic regime with high phylogenetic structuring of trait values 
$(a=5)$. We also assessed power as a function of the delay period before new species start interacting.

To evaluate the simulated data produced by the competition model, other comparative models were fitted to the data: the Brownian model itself; the $\delta$ model, which measures temporal acceleration and deceleration in rate; the $\kappa$ model, which measures the degree to which evolution is speciational rather than gradual (Pagel 1997); and $K$, a measure of phylogenetic signal (Blomberg et al. 2003). Parameter estimates were generated using the $\mathrm{R}$ packages geiger (Harmon et al. 2008) and picante (Kembel et al. 2010).

We compared two further common models to the competition model. Trait distributions and phylogenetic signal were computed for a single 25 -tip tree, using the competition model as well as the OU model (Hansen 1997) and the early burst (EB) model (Blomberg et al. 2003; Harmon et al. 2010a). Traits were simulated for the OU and EB models using the function $r$ TraitCont in the $\mathrm{R}$ package ape (Paradis et al. 2004). For the EB simulations, however, the tree is first time-transformed according to

$$
t \rightarrow \frac{e^{g t}-1}{g},
$$

where $t$ is time and $g$ parameterizes the model. When $g$ is negative, this represents a slowdown over time. As $g$ approaches 0 , the transformation approaches the identity, and we recover the Brownian model. We applied this transformation using the function transfBranchLengths in the $\mathrm{R}$ package phylolm (Ho and Ané 2014).

The simulations were written in $\mathrm{C}++$. Scripts for using these data sets for likelihood estimation were written in $\mathrm{R}$ (R Development Core Team 2015), using ape (Paradis et al. 2004) and TESS (Hoehna 2013) for tree generation (code is available online and also in GitHub [https://github.com /mcshef/treecomp/]).

\section{Case Study: Darwin's Finches}

The phylogeny of Galapagos finches was taken from Lamichhaney et al. (2015). We used a data set from Harmon et al. (2010b), using individual data originally from Lack (1947), with species values for five traits: wing length, tarsus length, bill length (culmen), bill depth, and bill width (gonys). We computed likelihood ratios for each trait individually as well as for combined pairs of beak traits. The final data sets used are deposited in the Dryad Digital Repository: http://dx.doi.org/10.5061/dryad.3sk15 (Clarke et al. 2017).

After simulating data on the phylogeny to determine likelihood cutoffs for rejecting BM, a likelihood comparison between the competition model and the nested BM model was run for each of the five traits separately. We performed the tests twice-once including and once excluding the phylogenetic summary statistic $K$ - to judge the importance of signal in favoring the competition model.

\section{Results}

\section{Example of Clade Evolution under the Competition Model}

Illustrative examples of evolution under the competition model are shown in figure 1. Estimates of phylogenetic signal $K$ and phenotypic rate change transformation parameters $\delta$ and $\kappa$ (Pagel 1997) are also shown for the simulated data. The evolution of each species is tracked through time from left to right. It can be seen that competition increases phylogenetic signal while giving the appearance of an overall slowdown in rates of trait evolution.

Competition tends to increase the overall variance in traits among the species in a phylogeny, as is clear from the increase in range and variation of traits moving from figure $1 \mathrm{~A}$ to figure $1 C$. This is because species experiencing competition from other species are more likely to evolve extreme trait values to become more different and escape competition.

As the strength of competition is increased, the differences between species become more clearly defined, with them occupying distinct positions in niche space. There are fewer intersections of traits' evolutionary paths over time between species, and the phylogenetic signal $K$ exceeds the neutral BM prediction of $K=1$. Competition thus increases phylogenetic signal above that expected under the $\mathrm{BM}$ model while presenting the appearance of a considerable tree-wide evolutionary slowdown. This slowdown can be seen in figure 1, where the rate change model $\delta$ is fitted to data sets simulated under competitive effects. This means that a species' trait values map more directly onto its position in the tree. For sympatric clades, there is thus a prediction of traits being more phylogenetically conserved than under BM.

Estimates for commonly used branch transformation parameter $\kappa$ from these data sets are also shown in figure 1. $\kappa$ measures the rate change along branches and overall measures the degree to which change is speciational (Pagel 1997). A transformation parameter $\delta$ models the overall changes in evolutionary rate across the tree, with lower values corresponding to evolutionary slowdowns (Pagel 1997). We find that the $\delta$ parameter diminishes very rapidly as competition is increased. It should be noted here that the $\delta$ parameter is biased such that the expectation values for $\mathrm{BM}$ are $>1$ (Freckleton et al. 2002). Nevertheless, this reflects an apparent slowdown of evolutionary rate, which becomes more pronounced as the value of $a$ increases. Species competing for unoccupied niche space thus evolve more rapidly early on in their development, when they are more similar to one another and the effects of competition are stronger, as one would expect in an adaptive radiation (Yoder et al. 2010). 
Figure $1 D$ shows a simulation using a combined competition and OU model (for details, see app. B). It can be seen that the repulsive effect of competition and the central attraction of the OU process have somewhat opposite effects, with competition tending to increase phylogenetic signal and rates of trait evolution, while the OU process reduces signal and the overall rate of trait change. This is part of the reason why we chose to base the competition model on the BM rather than the OU process. Even in real cases where both processes are present, the dominant process can likely be judged by the relative support for the competition and OU models considered separately. It seems unlikely that both components could be accurately fitted simultaneously, since their effects are so confounded.

Finally, we considered two cases of partial sympatry. In the first (fig. $1 E$ ), new lineages come into interaction with preexisting lineages only after a fixed delay period. This reduces phylogenetic signal relative to complete sympatry. The distribution of tip trait values is less affected, unless a substantial number of lineages remain in allopatry at the tree tips, that is, at the present time. The second case is analogous to two parallel adaptive radiations on isolated islands: all the descendants of one ancestral species interact with each other but not with the descendants of the second ancestral species. This removes the raised phylogenetic signal effect and most of the change in trait distribution. However, we can still detect competition, provided that we have a priori knowledge of the sympatry or allopatry of each species pair.

\section{Trait Distributions across Tree Tips}

The distribution of trait values of the phylogeny tips is flattened in the competition model compared with BM models, which predict normal distributions for large trees (fig. $2 A$ ). This outcome is expected when competition shapes trait values (Davies et al. 2012). The impact of competition on trait distributions is even more pronounced where hard limits are placed on the available range of trait values (also fig. 2A).

In addition to creating a more even trait distribution, competition increases the overall amount of trait divergence, given equal $\mathrm{BM}$ rates (fig. $2 \mathrm{~B}$ ). This is consistent with the expectation that equivalent species sets should be more diverged in sympatry than in allopatry when there is competition (Schluter 2000). From a biological perspective, there is thus a prediction that competition leads to a wider range of morphological variation in a clade, reflecting the increased tendency toward extreme traits when there is lots of competition.

\section{Effects of Tree Size}

We used trees normalized to the same total length, regardless of the number of tips. Given this normalization, under
$\mathrm{BM}$ and rate change models, the variance of tip trait values shows no change with increasing the number of tips (in agreement with Ricklefs [2004]). In the competition model, larger trees have greater variance, since a greater number of species are pushing each other away; this is shown in figure $2 C$. This relationship seems to be approximately linear for the unbounded competition model. When hard limits are imposed, the variance reaches a maximum corresponding to the positions of the extremes.

\section{Effects of Competition on Correlated Traits and Phylogenetic Signal}

For pairs of traits, in which the evolutionary changes in trait values are correlated, the correlation between the traits decays rapidly with increasing competition strength. This is even more pronounced when there are limits on extreme trait values. Figure $2 D$ and figure $2 E$ show how the correlation decays. By decorrelating traits, competition forces the trait space to be occupied more evenly.

Phylogenetic signal is increased by competition because species tend to remain adjacent in trait space to their close relatives (fig. $2 F$ ), and so their trait values are unlikely to cross over with time. Plots of traits through time therefore become more defined and tree-like. This can be seen, for example, in the sample simulations of figure 1. Correlation between traits has little effect on the phylogenetic signal exhibited by the individual traits under either the BM model or the competition model. Limits reduce the phylogenetic signal, since there is less trait space for distantly related species to diverge. Indeed, without competition driving the signal up (i.e., when $a=0$ ), the model with limits predicts reduced signal compared with the BM model, with $K$ estimated to be $<1$.

Price's (1997) model of adaptive radiations makes the unique prediction that when two traits have correlated evolution, the correlation between phylogenetically independent contrasts is potentially different in form than that between the traits themselves (Price 1997; Harvey and Rambaut 2000; Freckleton and Harvey 2006). In contrast, a Brownian model predicts equal correlation for both traits and contrasts.

We compared trait and contrast correlations under the competition model presented here. Competition tends to reduce correlation between traits, as discussed above, but we set the Brownian evolution of the traits to have very high correlation (fig. 3). We found that contrasts had higher correlations than traits. This probably reflects the fact that competition tends to have a greater effect earlier in the evolutionary history of any particular species. However, the general principle seems to hold, namely, that when species interact, the correlations between traits and trait changes (i.e., contrasts) are not expected to be equal. 

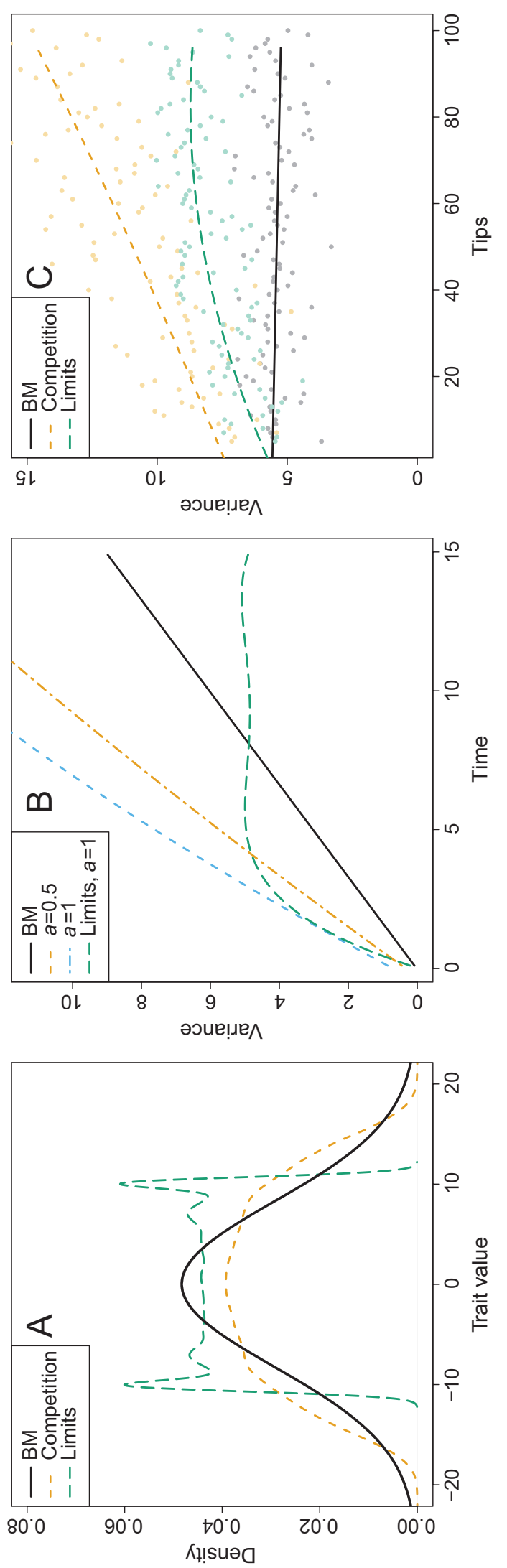
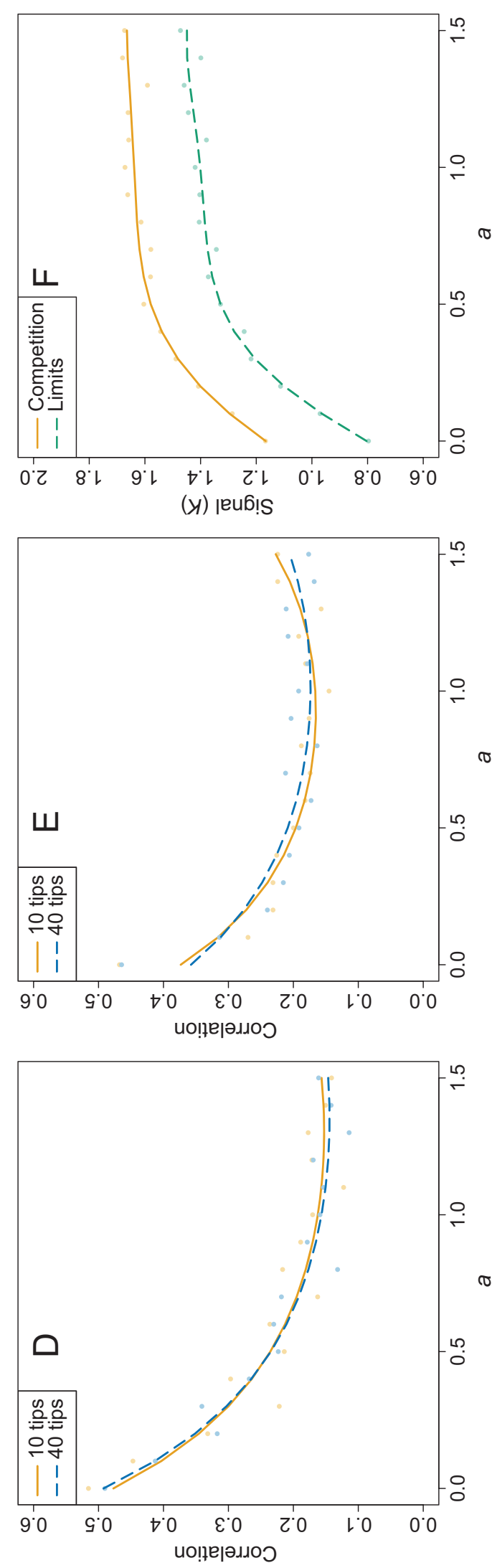

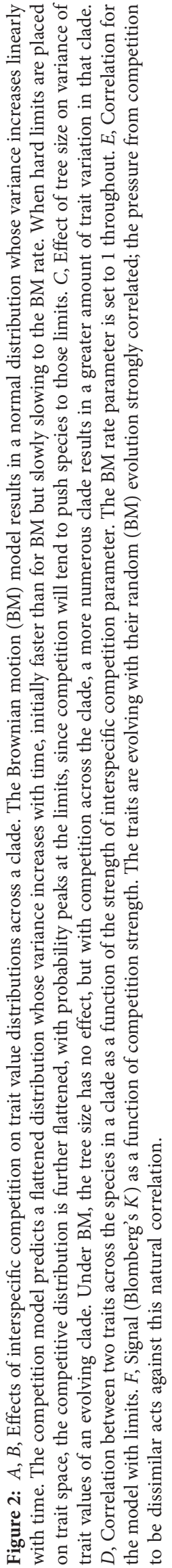

This content downloaded from 143.167.030.213 on January 17, 2017 04:14:20 AM 

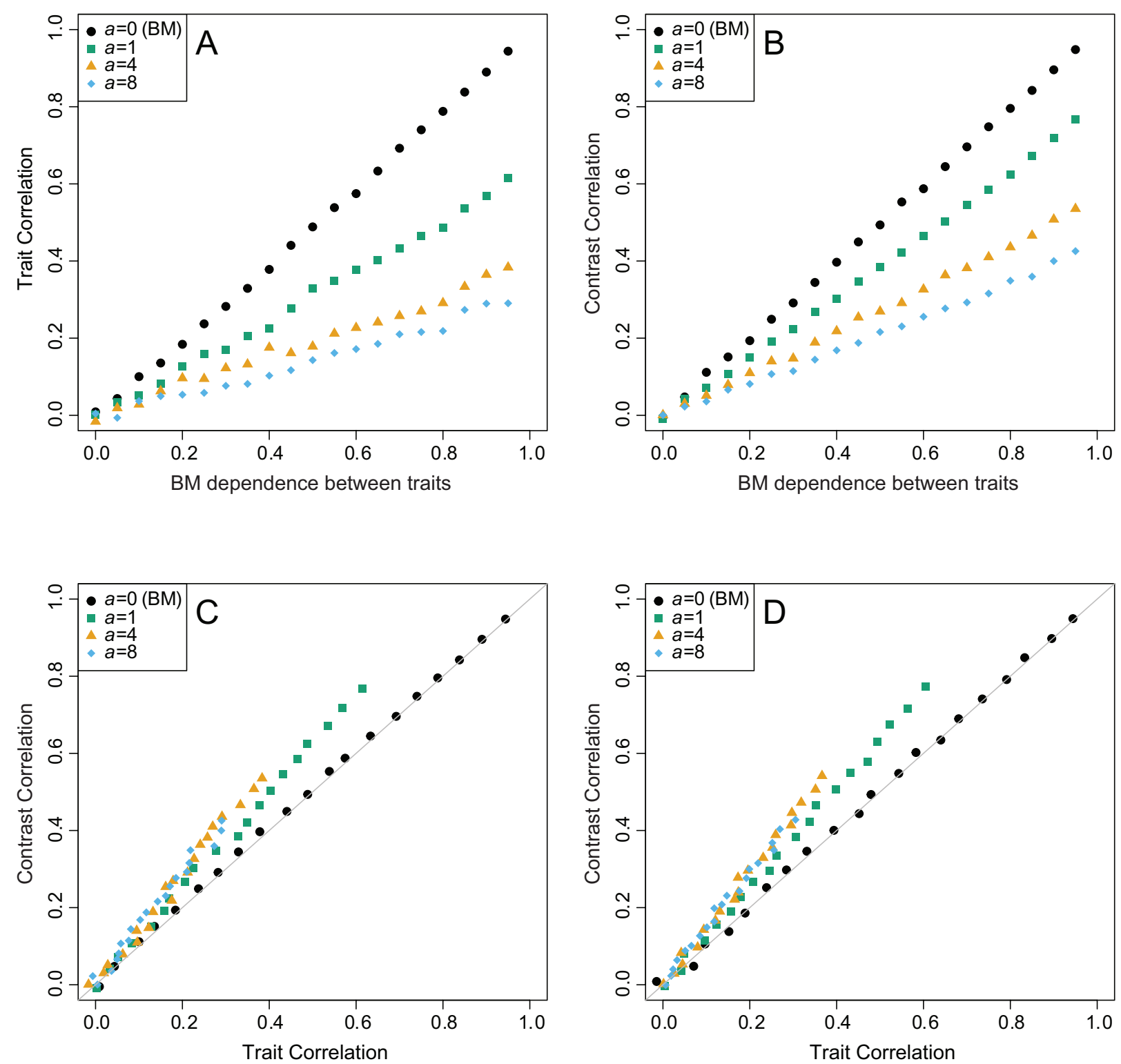

Figure 3: Traits and contrasts for two traits, where one trait has a dependence on the other. For each step in time, the dependent trait has an evolutionary change that depends on the change to the other trait. If the dependence is 1 , then these changes are equal; if the dependence is 0.5 , then the change in the dependent trait is 0.5 of the change in the other trait, and the remaining change is random. $A$, Trait correlation as a function of the intrinsic trait dependence. $B$, Contrast correlation as a function of the intrinsic trait dependence. $C$, Correlation between contrasts is slightly greater than correlation between traits for competitive evolution. $D$, Contrast and trait correlations for a model of competition with trait space limits.

\section{Comparisons with Alternative Models}

We generated trait distributions and computed phylogenetic signal for a single 25-tip tree, using the competition model as well as the OU model (Hansen 1997) and the EB model (Blomberg et al. 2003; Harmon et al. 2010a). (We in fact simulated on a range of trees, with sizes up to 100 tips, but report on a single tree here for the sake of com- parison. Tree size makes no qualitative difference to the observed patterns.) The expected distributions for $\mathrm{OU}$ and EB trait values remain normal, although the OU model reduces the variance relative to $\mathrm{BM}$. The actual trait distribution for a typically sized tree, however, was often multimodal for the EB model. In contrast, the competition model predicts a flattened, regular distribution of trait values. In this respect, the competition and $\mathrm{EB}$ predictions 
differ. Phylogenetic signal, on the other hand, is raised by both the competition model and the EB model, although more strongly by competition. The OU model reduces phylogenetic signal to lower values than expected under BM and to very low values when strong. These results are tabulated in appendix C (apps. C, D are available online).

\section{Power}

The power to detect competition effects against a background of BM evolution is shown in figure $4 A-4 D$ for trees of various sizes. We define the power as the frequency with which simulated data sets show significant support for competition effects as opposed to the (nested) BM null model. Power is greatest for large trees with high competition strength relative to $\mathrm{BM}$ rate. This can be interpreted as the relative contribution to overall evolutionary change of competitive effects versus other-effectively random - effects.

We also computed the power to distinguish data generated under a competition model from OU and EB models. This is shown in figure $4 F$. There is excellent power to detect even weak competition compared with these other models. This is not surprising, since a large amount of variation in trait distributions and phylogenetic signal can be generated under BM, and both the EB and the OU models represent deviations from $\mathrm{BM}$ that differ from the deviations caused by competition. Competition raises signal and reduces the variance in differences between neighboring tip trait values, whereas EB increases tip value difference variance, and OU reduces signal.

Figure $4 E$ shows power as a function of delay. Delay here means the time from a lineage originating to the time at which it starts interacting with all the preexisting lineages, measured as a proportion of the mean time between speciation events. While this delay reduces power, substantial power to detect strong competition remains even for delay periods of similar length to the time between speciation events.

In this context, a significant data set is one for which the type I error rate is estimated to be $\leq 0.05$. This is the frequency with which data from null model simulations display model likelihood ratios that equal or exceed the ratio for the observed data set. This is determined via a parametric bootstrap.

\section{Case Study: Darwin's Finches}

The simulations described above demonstrate two things: first, that the model we describe successfully captures behavior that we would expect to be observed in systems of interacting species; and second, that it may be applied to data and used to infer the presence of competitive interactions. In order to use the model in a real-world example, we applied the competition model to an example data set, using trait measurements collected by Harmon et al. (2010a; originally Grant and Grant 2002; Lack 1947; repository in
Harmon et al. 2010b) and a recent molecular phylogeny (Lamichhaney et al. 2015). We used the Galapagos finches (Geospiza spp.) because they are a well-studied adaptive radiation, and ecological effects were anticipated to be of importance. The effect of character displacement on intraspecific variation among these finches is well documented (e.g., Grant and Grant 2006). Here we are looking to see whether evidence for this mechanism can be detected in the overall distribution of traits across the clade.

The parameter estimates and model likelihood ratios are shown in table 1 . The beak traits showed greater support for competition compared with Brownian evolution than the other traits. This appears to point to an ecological effect: the competition model implies a tendency toward well-differentiated niches that do not cross, and the beak shape is an ecological trait, in the sense that it corresponds strongly to feeding habits (Grant and Grant 2011). Multitrait analyses for pairs of beak traits are also shown in table 1. Competition tends to reduce correlation between traits in our model, however (see fig. 1). Since beak measurements are likely to be correlated (because of overall size), the model may have a poor fit without adding correlated Brownian evolution as a further fitted parameter, which we have not done. The model as it stands may be better suited to principle component data, where correlations have already been accounted for and removed before the analysis. Figure 4 shows illustrative plots of simulated trait evolution, using the model parameters that were estimated for the culmen length. Compared with $\mathrm{BM}$, shown in figure $4 A$, the tree becomes very well defined, with strong phylogenetic signal.

One of the beak traits (culmen length) favored the competition model when signal was not used but less so when it was included. Brownian rate parameter estimates under the model with limits are higher than those for the nonlimited competition model. This higher rate does not result in a greater total amount of evolutionary change because of the hard limits that are reached either way. This result does, however, suggest that the niche landscape may be the limiting factor in the finches' evolution: the finch traits are capable of evolving rapidly, but their values are constrained by the combination of interactions between species and environmental limits on niche space.

It is worth noting that none of these results takes into account intraspecific variation or uncertainty in the finch phylogeny. Species interactions will be somewhat independent on different islands, as illustrated by the character displacement seen in intraspecific trait variation (Grant and Grant 2006).

\section{Discussion}

There have been several recent approaches to integrating ecological processes into phylogenetic models of evolution 

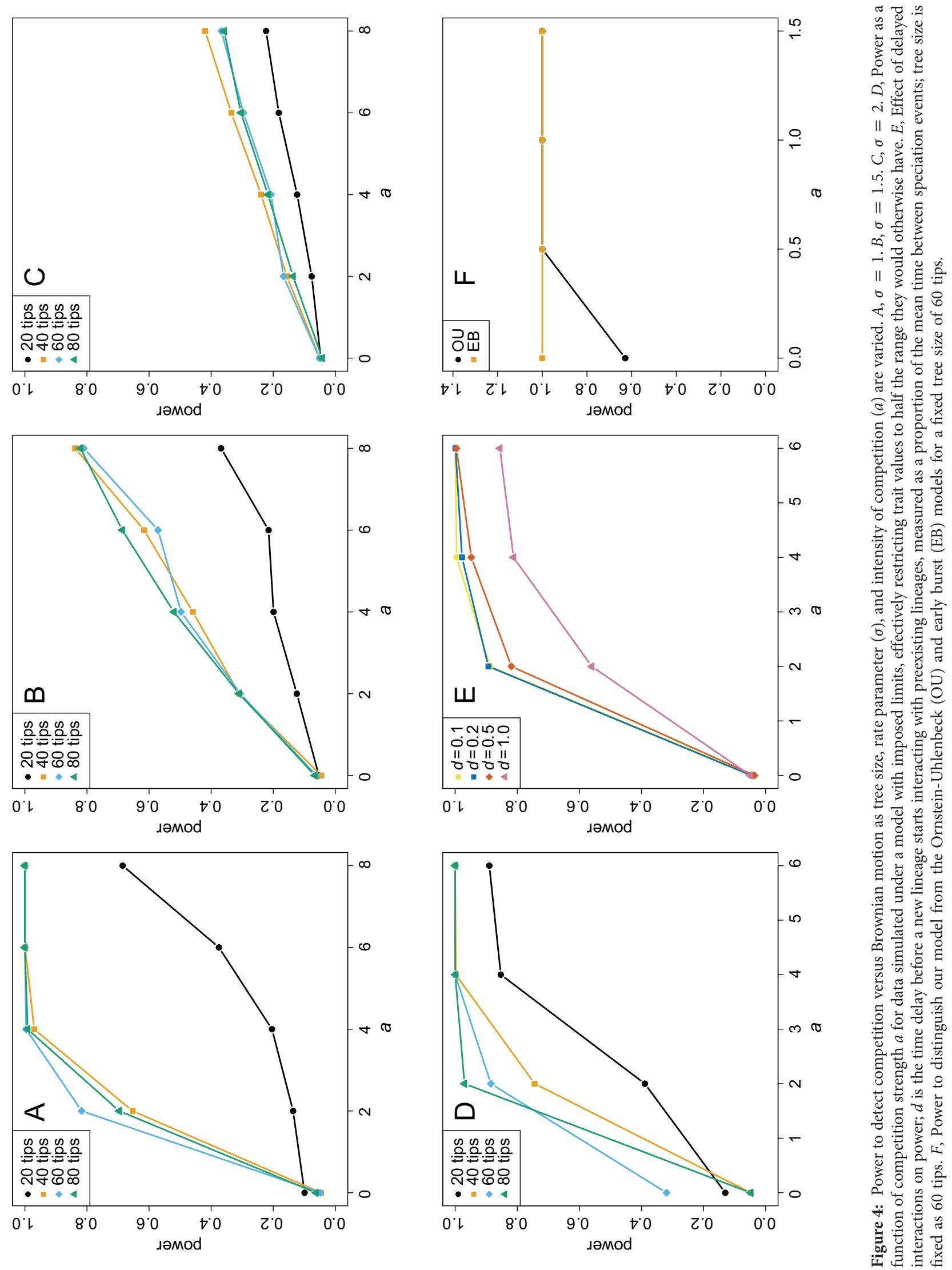

This content downloaded from 143.167.030.213 on January 17, 2017 04:14:20 AM 
Table 1: Traits and likelihood ratio test statistics (LRTS) for model comparisons for Galapagos finches

\begin{tabular}{lccc}
\hline Trait & $\sigma$ & $a$ & LRTS \\
\hline Wing length & 1.48 & .28 & 1.01 \\
Tarsus length & 1.64 & .48 & 1.04 \\
Culmen length & 1.24 & .96 & $4.34^{*}$ \\
Beak depth & 1.40 & 1.92 & 2.73 \\
Gonys width & 1.48 & 1.32 & 1.87 \\
Culmen + beak depth & 1.12 & 3.76 & 3.11 \\
Culmen + gonys width & 1.12 & 4.20 & 3.24 \\
\hline
\end{tabular}

Note: The finch trait data set is that given by Harmon et al. (2010b). The competition model is compared with the nested Brownian motion (BM) model. The competition model has one extra parameter compared with the BM model.

* Significance based on simulated parameter distributions.

(Cavender-Bares et al. 2009; Pennell and Harmon 2013; Hadfield et al. 2014). As a step in this direction, we have created a model of interspecific competition on phylogenies of coexisting species. The model provides a processbased picture of competitive evolution, linking statistical patterns directly to the underlying ecology. It generates the patterns we expect to see in situations where interspecific competition is important.

Competition and niche overlap have a complex relationship. According to one scenario, niches form a continuum, with similar fitnesses along a niche axis. Species compete for empty regions of the niche axis and evolve minimally overlapping, evenly spaced trait values, consistent with the ecological idea of character displacement (Grant 1972; Strong et al. 1979; Dayan and Simberloff 2005). Our model accommodates this process in a phylogenetic context. However, according to a second scenario, there is an optimum position on a niche axis, and species will compete with each other to occupy it. Consequently, they will evolve similar trait values tailored to that optimum (Colwell and Futuyma 1971), a form of convergent evolution. Traits that have evolved like this are likely to be poorly represented by our competition model, and we would be unlikely to find evidence supporting the model. Instead, such a process would be better modeled by a process such as an OU model (Hansen 1997; Butler and King 2004). The model we have introduced here corresponds better to a process of occupancy of distinct niches distributed across trait space.

One prediction of the competition model is a flattened distribution of trait values among contemporary species of a single sympatric clade. Indeed, competition is often inferred from such evenness (Dayan and Simberloff 2005; Davies et al. 2012). The same pattern can, however, be caused by competition at the community assembly level rather than in situ trait evolution (Cavender-Bares et al. 2009; Stuart and Losos 2013) or by geographical structure in speciation and extinction (Pigot and Etienne 2015). For this reason, the model presented here is best applied to complete clades rather than local paraphyletic communities. Since complete clades are rarely entirely sympatric, we have included the ability to turn competitive interactions on and off on a pairwise basis, depending on sympatry. The model can similarly include a delay between speciation events and the start of competitive interactions involving the new species, corresponding to allopatric speciation and subsequent range expansion. This is important since there is a wealth of variation between clades in species' geographic structure and opportunity to interact (Fitzpatrick et al. 2008). Strong niche conservatism is, nevertheless, predicted for largely sympatric clades. Developing summary statistics for testing our model on data with substantial allopatry - for example, multiple island radiations - will be an important future development.

The pattern of non-Brownian trait distributions and high phylogenetic signal is also generated by an alternative but related mechanism, where - instead of there being a continuum of possible niches - the niches are discrete and new species arise by jumping to a nearby niche (Price 1997; Harvey and Rambaut 2000; Freckleton and Harvey 2006). In these models, niches appear at random, and existing species that are nearby in niche space can speciate to occupy a new niche. Determining a method to distinguish this type of model from diffusion models will be a useful future development. Specifically, this approach differs from the other models discussed here in that the tree topology is not fixed but interacts with species' trait values as the clade evolves.

There are numerous speciation/extinction models for phylogenies (Nee et al. 1994; Pybus and Harvey 2000; Rabosky 2006; Freckleton et al. 2008; FitzJohn 2010), including some that are expected to correspond to clades with interspecific competition (Harmon et al. 2010a; Etienne et al. 2012). Our model is concerned only with trait evolution. Trait evolution and diversification rates may be coupled in nature, however, and both may vary with factors such as interspecific competition. Building models of adaptive radiations that simultaneously predict trait evolution and diversification will be key in the future.

Most phylogenetic models of trait evolution are modifications of the random BM model. As noted above, adaptive radiations are generally consistent with a tree-wide gradual slowdown in rates of phenotypic evolution ( $\delta$ model: Pagel 1997; ACDC model: Blomberg et al. 2003). Speciational evolution can be modeled as a gradual branch-wise slowdown ( $\kappa$ model: Pagel 1997$)$ or by partitioning evolution into gradual and speciational parts (Bokma 2008; Ingram 2010). Discrete shifts in evolutionary rate can be modeled to detect, for example, adaptive radiations embedded in a larger tree (O'Meara et al. 2006; Thomas et al. 2006). Slowdowns in evolutionary rate have also been observed as a function not of time but directly of a clade's size (Mahler et al. 2010). The 
results for our competition model suggest that it reproduces the appearance of a strong tree-wide slowdown. During a radiation, though, competition is predicted to cause overall trait variance to increase much more rapidly. Our results for the competition model also demonstrate raised phylogenetic signal when most species are sympatric and have opportunity to interact, in agreement with similar results from Nuismer and Harmon (2015).

In all analyses, we used a fixed competition kernel width. The fact that this width is not distinguishable from the competition strength itself suggests that the amount of variation possible within a single niche is not readily ascertained from a phylogeny and trait data. Measurements of intraspecific variation will be more suited to this question. In fact, the competition kernel widths could be set empirically before analysis, if data on intraspecific variation were available.

Our results for the Galapagos finches support the wellknown presence of character displacement in that clade (Grant and Grant 2006) and further suggest that interspecific competition is a significant force comparable with othereffectively random - sources of evolutionary change for the Galapagos finches. For some beak measurements, the Galapagos finches exhibit the elevated phylogenetic signal predicted by the competition model, and for beak length, we find strong support for the model.

As phylogenetic methods continue to be used to infer evolutionary processes, it will be important to include specific ecological mechanisms (Vamosi et al. 2009). Competition for ecologically distinct roles is often implicitly or explicitly assumed in adaptive radiations, but its prevalence and importance remain uncertain (Schluter 2000; Stuart and Losos 2013). We have developed an explicit model of competition on phylogenies to detect competitive effects in sympatric adaptive radiations and to enable measurement of competition strength. The predictions of this model may help in understanding the roles ecological processes play in shaping trait evolution.

\section{Acknowledgments}

We thank N. Cooper and two anonymous reviewers for extensive useful feedback on the manuscript. G.H.T. was supported by the Natural Environment Research Council (grant NE/G012938/1).

\section{APPENDIX A}

\section{Estimating Competition Strength and} Effect Width Simultaneously

The overlap between species $i$ and $j$ is proportional to $\Phi\left(-\Delta X_{i j} / 2 \sigma\right)$, where $\Phi$ is the cumulative normal distribu- tion. Integrating by parts yields the following approximate function for the overlap between two species:

$$
\begin{aligned}
\Phi\left(\frac{-\left|\Delta X_{i j}\right|}{2 \sigma}\right)= & 0.5-\frac{1}{\sqrt{2 \pi}} e^{\left(-\left|\Delta X_{i j}\right|^{2} / /\left(8 \sigma^{2}\right)\right.} \\
& \left(\frac{\left|\Delta X_{i j}\right|}{2 \sigma}+\frac{\left|\Delta X_{i j}\right|^{3}}{24 \sigma^{3}}+\cdots\right) .
\end{aligned}
$$

To obtain the evolutionary rate, we multiply this by $a$, giving

$$
\begin{aligned}
a \Phi\left(\frac{-\left|\Delta X_{i j}\right|}{2 \sigma}\right)= & 0.5 a-\frac{a}{\sqrt{2 \pi}} e^{\left(-\left|\Delta X_{i j}\right|^{2}\right) /\left(8 \sigma^{2}\right)} \\
& \left(\frac{\left|\Delta X_{i j}\right|}{2 \sigma}+\frac{\left|\Delta X_{i j}\right|^{3}}{24 \sigma^{3}}+\cdots\right) .
\end{aligned}
$$

At first glance, it might appear that changing $a$ and $\sigma$ would have different effects because the former changes evolutionary rates in a linear manner, while the effect of the latter is nonlinear. However, if there are a large number of species within a limited niche space, then distances between species will be low; that is, $\left|\Delta X_{i j}\right|$ is small. Consequently, we can use the following approximation by the Maclaurin series expansion of $e^{x}$ :

$$
e^{\left(-\left|\Delta X_{i j}\right|^{2}\right) /\left(8 \sigma^{2}\right)} \approx 1-\frac{1}{8 \sigma^{2}}\left|\Delta X_{i j}\right|^{2} .
$$

Substituting into equation (A2) and ignoring higher than squared terms, we obtain

$$
\begin{aligned}
& a \Phi\left(\frac{-\left|\Delta X_{i j}\right|}{2 \sigma}\right) \approx 0.5 a-\frac{a}{\sqrt{2 \pi}}\left(1-\frac{1}{8 \sigma^{2}}\left|\Delta X_{i j}\right|^{2}\right) \\
&\left(\frac{\left|\Delta X_{i j}\right|}{2 \sigma}\right) \approx 0.5 a\left(1-\frac{\left|\Delta X_{i j}\right|}{\sigma \sqrt{2 \pi}}\right) .
\end{aligned}
$$

Overall, the rate of evolution is given by the overlap, $2 \Phi\left(-\Delta X_{i j} / 2 \sigma\right)$, multiplied by $a$, yielding

$$
2 a \Phi\left(\frac{-\left|\Delta X_{i j}\right|}{2 \sigma}\right) \approx a\left(1-\frac{\left|\Delta X_{i j}\right|}{\sigma \sqrt{2 \pi}}\right) .
$$

This equation is linear in both $a$ and $\sigma^{-1}$. Thus, from a statistical perspective, $a$ and $\sigma$ will be nonidentifiable if the species are interacting strongly. If species are not interacting strongly - that is, $\Delta X_{i j}$ is large - then the data will contain no information on interactions between species; hence, it will not be possible to fit the model, and we cannot estimate either $a$ or $\sigma$.

\section{APPENDIX B}

\section{Ornstein-Urhlenbeck Model with Competition}

We based our competition model on BM for reasons detailed in the introduction. However, for the sake of com- 
parison, we also simulated under a model that included both OU and character displacement processes. The result is random diffusion with attraction of all lineages to a central optimum but repulsion between lineages.

The instantaneous change in the OU model of the trait value $x$ for any given lineage is given in differential form by

$$
\mathrm{d} x(t)=-\alpha(x(t)-\psi)+\sigma \mathrm{d} W_{i}(t),
$$

where $\psi$ represents the optimum trait value to which lineages are attracted. The OU + competition model combines equation (3) and equation (B1), giving

$$
\begin{aligned}
\mathrm{d} x_{i}= & -\alpha(x(t)-\psi) \\
& +a \sum_{j} S_{i j}(t) e_{i j} 2 \Phi\left(-\left|x_{i}-x_{j}\right|\right) \mathrm{d} t+\sigma \mathrm{d} W_{i}(t) .
\end{aligned}
$$

\section{Literature Cited}

Bartoszek, K., J. Pienaar, P. Mostad, S. Andersson, and T. F. Hansen. 2012. A phylogenetic comparative method for studying multivariate adaptation. Lournal of Theoretical Biology 314:204-215.

Beaumont, M. A. 2010. Approximate Bayesian computation in evolution and ecology. Annual Review of Ecology, Evolution, and Systematics 41:379-406.

Blomberg, S. P., T. Garland, and A. R. Ives. 2003. Testing for phylogenetic signal in comparative data: behavioural traits are more labile. Evolution 57:717-745.

Bokma, F. 2008. Detection of "punctuated equilibrium" by Bayesian estimation of speciation and extinction rates, ancestral character states, and rates of anagenetic and cladogenetic evolution on a molecular phylogeny. Evolution 62:2718-2726.

Boucher, F. C., and V. Démery. 2016. Inferring bounded evolution in phenotypic characters from phylogenetic comparative data. Systematic Biology 65:651-661.

Butler, M. A., and A. A. King. 2004. Phylogenetic comparative analysis: a modeling approach for adaptive evolution. American Naturalist 164:683-695.

Cavender-Bares, J., K. H. Kozak, P. V. A. Fine, and S. W. Kembel. 2009. The merging of community ecology and phylogenetic biology. Ecology Letters 12:693-715.

Clarke, M., Freckleton, R. P., and G. H. Thomas. 2017. Data from: Trait evolution in adaptive radiations: modeling and measuring interspecific competition on phylogenies. American Naturalist, Dryad Digital Repository, http://dx.doi.org/10.5061/dryad.3sk15.

Colwell, R. K., and D. J. Futuyma. 1971. On the measurement of niche breadth and overlap. Ecology 52:567-576.

Csilléry, K., M. G. B. Blum, O. E. Gaggiotti, and O. François. 2010 Approximate Bayesian computation (ABC) in practice. Trends in Ecology and Evolution 7:410-408.

Davies, T. J., N. Cooper, J. A. F. Diniz-Filho, G. H. Thomas, and S. Meiri. 2012. Using phylogenetic trees to test for character displacement: a model and an example from a desert mammal community. Ecology 93:S44-S51.

Dayan, T., and D. Simberloff. 2005. Ecological and community-wide character displacement: the next generation. Ecology Letters 8:875894.
De Mazancourt, C., E. Johnson, and T. G. Barraclough. 2008. Biodiversity inhibits species' evolutionary responses to changing environments. Ecology Letters 11:380-388.

Doebeli, M., and U. Dieckmann. 2003. Speciation along environmental gradients. Nature 421:259-264.

Eastman, J. M., M. E. Alfaro, P. Joyce, A. L. Hipp, and L. J. Harmon. 2011. A novel comparative method for identifying shifts in the rate of character evolution on trees. Evolution 65:3578-3589.

Emerson, B. C., and R. G. Gillespie. 2008. Phylogenetic analysis of community assembly and structure over space and time. Trends in Ecology and Evolution 23:619-630.

Etienne, R. S., B. Haegeman, T. Stadler, T. Aze, P. N. Pearson, A. Purvis, and A. B. Phillimore. 2012. Diversity-dependence brings molecular phylogenies closer to agreement with the fossil record. Proceedings of the Roval Society B 279:1300-1309.

Felsenstein, J. 1973. Maximum-likelihood estimation of evolutionary trees from continuous characters. American Journal of Human Genetics 25:471-492.

1985. Phylogenies and the comparative method. American Naturalist 125:1-15.

FitzJohn, R. G. 2010. Quantitative traits and diversification. Systematic Biology 59:619-633.

Fitzpatrick, B. M., J. A. Fordyce, and S. Gavrilets. 2008. What, if anything, is sympatric speciation? Journal of Evolutionary Biology 21: 1452-1459.

Freckleton, R. P. 2009. The seven deadly sins of comparative analysis. Џournal of Evolutionary Biology 22:1367-1375.

Freckleton, R. P., and P. H. Harvey. 2006. Detecting non-Brownian trait evolution in adaptive radiations. PLoS Biology 4:e373.

Freckleton, R. P., P. H. Harvey, and M. Pagel. 2002. Phylogenetic analysis and comparative data: a test and review of evidence. American Naturalist 160:712-726.

Freckleton, R. P., M. Pagel, and P. H. Harvey. 2003. Comparative methods for adaptive radiations. Pages 391-407 in T. M. Blackburn and K. J. Gaston, eds. Macroecology: concepts and consequences. Blackwell Scientific, Oxford.

Freckleton, R. P., A. B. Phillimore, and M. Pagel. 2008. Relating traits to diversification: a simple test. American Naturalist 172:102-115.

Garland, T., P. H. Harvey, and A. R. Ives. 1992. Procedures for the analysis of comparative data using phylogenetically independent contrasts. Systematic Biology 41:18-32.

Gillespie, R. G., F. G. Howarth, and G. K. Roderick. 2001. Adaptive radiation. Encyclopedia of Biodiversity 1:25-44.

Grant, P. R. 1972. Convergent and divergent character displacement. Biological Journal of the Linnean Society 4:39-68.

Grant, P. R., and B. R. Grant. 2002. Unpredictable evolution in a 30year study of Darwin's finches. Science 296:707-711.

. 2006. Evolution of character displacement in Darwin's finches. Science 313:224-226

2011. How and why species multiply: the radiation of Darwin's finches. Princeton University Press, Princeton, NJ.

Hadfield, J. D., B. R. Krasnov, R. Poulin, and S. Nakagawa. 2014. A tale of two phylogenies: comparative analyses of ecological interactions. American Naturalist 183:174-187.

Hansen, T. F. 1997. Stabilizing selection and the comparative analysis of adaptation. Evolution 51:1341-1351.

Harmon, L. J., J. B. Losos, T. J. Davies, R. G. Gillespie, J. L. Gittleman, W. B. Jennings, K. H. Kozak, et al. 2010a. Early bursts of body size and shape evolution are rare in comparative data. Evolution 64:2385-2396. 
. 2010b. Data from: Early bursts of body size and shape evolution are rare in comparative data. Evolution 64:2385-2396, Dryad Digital Repository, http://dx.doi.org/10.5061/dryad.f660p.

Harmon, L. J., J. T. Weir, C. D. Brock, R. E. Glor, and W. Challenger. 2008. GEIGER: investigating evolutionary radiations. Bioinformatics 24:129-131.

Hartig, F., J. M. Calabrese, B. Reineking, T. Wiegand, and A. Huth. 2011. Statistical inference for stochastic simulation models - theory and application. Ecology Letters 14:816-827.

Harvey, P. H., and A. Rambaut. 2000. Comparative analyses for adaptive radiations. Philosophical Transactions of the Roval Society B 355:1599-1605.

Ho, L. S. T., and C. Ané. 2014. A linear-time algorithm for Gaussian and non-Gaussian trait evolution models. Systematic Biology 63 397-408.

Hoehna, S. 2013. Fast simulation of reconstructed phylogenies under global, time-dependent birth-death processes. Bioinformatics 29 : $1367-1374$.

Ingram, T. 2010. Speciation along a depth gradient in a marine adaptive radiation. Proceedings of the Roval Society B 278:613-618.

Inman, H. F., and E. L. Bradley. 1989. The overlapping coefficient as a measure of agreement between probability distributions and point estimation of the overlap of two normal densities. Communications in Statistics: Theory and Methods 18:3851-3874.

Johnson, M. T. J., and J. R. Stinchcombe. 2007. An emerging synthesis between community ecology and evolutionary biology. Trends in Ecology and Evolution 22:250-257.

Jungck, J. R. 2001. BioQUEST library. Vol. 6. Academic Press, Cambridge, MA.

Kembel, S. W., P. D. Cowan, M. R. Helmus, W. K. Cornwell, H. Morlon, D. D. Ackerly, S. P. Blomberg, and C. O. Webb. 2010 Picante: R tools for integrating phylogenies and ecology. Bioinformatics 26:1463-1464.

Kraft, N. J. B., W. K. Cornwell, C. O. Webb, and D. D. Ackerly. 2007. Trait evolution, community assembly, and the phylogenetic structure of ecological communities. American Naturalist 170:271283.

Kutsukake, N., and H. Innan. 2013. Simulation-based likelihood approach for evolutionary models of phenotypic traits on phylogeny. Evolution 67:355-367.

Lack, D. 1947. The significance of clutch size. Ibis 89:302-352.

Lamichhaney, S., J. Berglund, M. S. Almén, K. Maqbool, M. Grabherr, A. Martinez-Barrio, M. Promerová, et al. 2015. Evolution of Darwin's finches and their beaks revealed by genome sequencing. $\mathrm{Na}-$ ture 518:371-375.

Leimar, O., M. Doebeli, and U. Dieckmann. 2008. Evolution of phenotypic clusters through competition and local adaptation along an environmental gradient. Evolution 62:807-822.

Leimar, O., A. Sasaki, M. Doebeli, and U. Dieckmann. 2013. Limiting similarity, species packing, and the shape of competition kernels. Lournal of Theoretical Biology 339:3-13.

Mahler, D. L., L. J. Revell, R. E. Glor, and J. B. Losos. 2010. Ecological opportunity and the rate of morphological evolution in the diversification of greater antillean anoles. Evolution 64:2731-2745.

Mayfield, M. M., and J. M. Levine. 2010. Opposing effects of competitive exclusion on the phylogenetic structure of communities. Ecology Letters 13:1085-1093.

Moen, D. S., and J. J. Wiens. 2009. Phylogenetic evidence for competitively driven divergence: body-size evolution in Caribbean treefrogs (Hylidae: Osteopilus). Evolution 63:195-214.
Nee, S., E. C. Holmes, R. M. May, and P. H. Harvey. 1994. Extinction rates can be estimated from molecular phylogenies. Philosophical Transactions of the Roval Society B 344:77-82.

Nuismer, S. L., and L. J. Harmon. 2015. Predicting rates of interspecific interaction from phylogenetic trees. Ecology Letters 18:17-27.

O'Meara, B. C., C. Ané, M. J. Sanderson, and P. C. Wainwright. 2006. Testing for different rates of continuous trait evolution using likelihood. International Journal of Organic Evolution 60:922-933.

Pagel, M. 1997. Inferring evolutionary processes from phylogenies. Zoologica Scripta 26:331-348.

Paradis, E., J. Claude, and K. Strimmer. 2004. APE: analyses of phylogenetics and evolution in R language. Bioinformatics 20:289-290.

Pennell, M. W., and L. J. Harmon. 2013. An integrative view of phylogenetic comparative methods: connections to population genetics, community ecology, and paleobiology. Annals of the New York Academy of Sciences 1289:90-105.

Pfennig, D. W., and K. S. Pfennig. 2010. Character displacement and the origins of diversity. American Naturalist 176(suppl.):S26-S44.

Pigolotti, S., C. López, E. Hernández-García, and K. H. Andersen. 2010. How Gaussian competition leads to lumpy or uniform species distributions. Theoretical Ecology 3:89-96.

Pigot, A. L., and R. S. Etienne. 2015. A new dynamic null model for phylogenetic community structure. Ecology Letters 18:153-163.

Price, T. D. 1997. Correlated evolution and independent contrasts. Philosophical Transactions of the Roval Society B 352:519-529.

Price, T. D., D. M. Hooper, C. D. Buchanan, U. S. Johansson, D. T. Tietze, P. Alström, U. Olsson, et al. 2014. Niche filling slows the diversification of Himalayan songbirds. Nature 509:222-225.

Pybus, O. G., and P. H. Harvey. 2000. Testing macro-evolutionary models using incomplete molecular phylogenies. Proceedings of the Roval Societv B 267:2267-2272.

Rabosky, D. L. 2006. Likelihood methods for detecting temporal shifts in diversification rates. Evolution 60:1152-1164.

R Development Core Team. 2015. R: a language and environment for statistical computing. R Foundation for Statistical Computing, Vienna. https://www.R-project.org/.

Revell, L. J., D. L. Mahler, P. R. Peres-Neto, and B. D. Redelings. 2012. A new phylogenetic method for identifying exceptional phenotypic diversification. Evolution 66:135-146.

Ricklefs, R. E. 2004. Evolution: how do characters evolve? (reply). Nature 432, doi:10.1038/nature03092.

Schluter, D. 2000. Ecological character displacement in adaptive radiation. American Naturalist 156(suppl.):S4-S16.

Schluter, D., and J. D. McPhail. 1992. Ecological character displacement and speciation in sticklebacks. American Naturalist 140:85-108.

Schoener, T. W. 2011. The newest synthesis: understanding the interplay of evolutionary and ecological dynamics. Science 331:426-429.

Slater, G. J., L. J. Harmon, D. Wegmann, P. Joyce, L. J. Revell, and M. E. Alfaro. 2012. Fitting models of continuous trait evolution to incompletely sampled comparative data using approximate Bayesian computation. Evolution 66:752-762.

Snodgrass, R. E., and E. Heller. 1904. Papers from the HopkinsStanford Galápagos Expedition, 1898-1899. Proceedings of the Washington Academy of Sciences 5:231-372.

Strong, D. R., Jr., L. A. Szyska, and D. S. Simberloff. 1979. Test of community-wide character displacement against null hypotheses. Evolution 33:897-913.

Stuart, Y. E., and J. B. Losos. 2013. Ecological character displacement: glass half full or half empty? Trends in Ecology and Evolution 28: 402-408. 
Swarth, H. S. 1931. The avifauna of the Galapagos islands. California Academy of Sciences 18, San Francisco.

Thomas, G. H., and R. P. Freckleton. 2012. MOTMOT: models of trait macroevolution on trees. Methods in Ecology and Evolution 3:145-151.

Thomas, G. H., R. P. Freckleton, and T. Székely. 2006. Comparative analyses of the influence of developmental mode on phenotypic diversification rates in shorebirds. Proceedings of the Roval Society B 273:1619-1624.

Uyeda, J. C., and L. J. Harmon. 2014. A novel Bayesian method for inferring and interpreting the dynamics of adaptive landscapes from phylogenetic comparative data. Systematic Biology 63:902918.
Vamosi, S. M., S. B. Heard, J. C. Vamosi, and C. O. Webb. 2009 Emerging patterns in the comparative analysis of phylogenetic community structure. Molecular Ecology 18:572-592.

Webb, C. O., D. D. Ackerly, M. A. McPeek, and M. J. Donoghue. 2002. Phylogenies and community ecology. Annual Review of Ecology and Systematics 33:475-505.

Yoder, J. B., E. Clancey, S. Des Roches, J. M. Eastman, L. Gentry, W. Godsoe, T. J. Hagey, et al. 2010. Ecological opportunity and the origin of adaptive radiations. Journal of Evolutionary Biology 23: 1581-1596.

Associate Editor: Scott J. Steppan Editor: Judith L. Bronstein

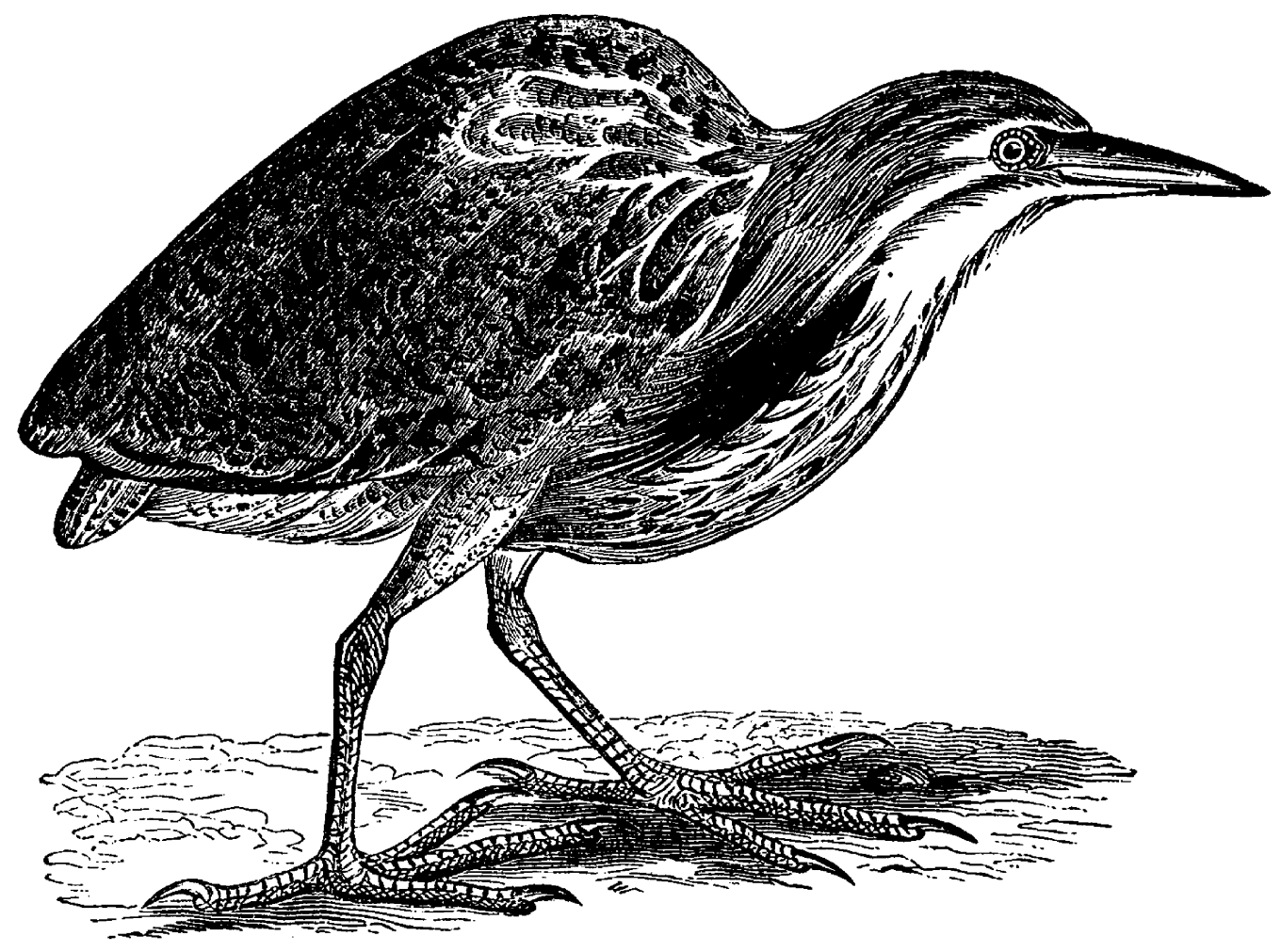

"In old times the bittern was held in high esteem for the sport it afforded when pursued by trained falcons. Both birds would mount in spirals, oftentimes out of sight; the bittern straining every nerve to keep above the hawk, the hawk doing his best to rise above the bittern so as to make the fatal pounce. The bittern, being of weaker flight, rarely escaped, but often in his death involved his enemy's; for as the cruel falcon came down with rushing wings, exulting in his fierce soul, the bittern, in his dire extremity, thrusting up his sharp beak, empaled the triumphant savage, and both came tumbling from the clouds together, striking the earth with a thump which drove the last breath from both. A lesson to tyrants not to push the weak to despair." From "Bitterns" by William E. Endicott (The American Naturalist 1869, 3:169-179). 